\title{
Didactiser la tension narrative : apprendre à lire ou apprendre comment le récit nous fait lire?
}

\section{Raphaël Baroni}

\section{Q OpenEdition}

12 Journals

Édition électronique

URL : http://journals.openedition.org/recherchestravaux/649

DOI : 10.4000/recherchestravaux.649

ISSN : 1969-6434

Éditeur

UGA Éditions/Université Grenoble Alpes

\section{Édition imprimée}

Date de publication : 31 décembre 2013

Pagination : 11-24

ISBN : 978-2-84310-267-7

ISSN : 0151-1874

\section{Référence électronique}

Raphaël Baroni, « Didactiser la tension narrative : apprendre à lire ou apprendre comment le récit nous fait lire? », Recherches \& Travaux [En ligne], 83 | 2013, mis en ligne le 01 juillet 2015, consulté le 08 septembre 2020. URL : http://journals.openedition.org/recherchestravaux/649 ; DOI : https://doi.org/ 10.4000/recherchestravaux.649 


\section{Didactiser la tension narrative : apprendre à lire ou apprendre comment le récit nous fait lire?}

Dans ce chapitre, j'essaierai d'articuler la notion de tension narrative - sur laquelle je travaille depuis une douzaine d'années dans le champ de la théorie du récit - avec les questions relatives à la lecture et à l'enseignement de la littérature. À première vue, cet effet de lecture, que l'on peut associer au suspense ou à la curiosité que nous inspirent des intrigues bien nouées, semble doublement incompatible avec la lecture telle qu'elle est ordinairement pratiquée et valorisée dans l'institution scolaire. En effet, l'enseignement de la littérature se donne souvent pour mission d'être le lieu d'un apprentissage de la lecture nécessitant de sortir de l'immersion, de dépasser l'illusion mimétique et de se désintéresser de l'intrigue, de la psychologie des personnages ou du pathos en général. D'ailleurs, il va sans dire que nous n'avons guère besoin de recevoir un enseignement spécifique pour apprendre à lire pour l'intrigue, puisque, dès notre plus jeune âge, nous sommes capables de réagir aux ressorts narratifs et de jouir du suspense qui nous étreint lorsque les aventures du Petit Tailleur ou de la Petite Sirène se compliquent. De plus, la tension narrative possède le défaut paradoxal d'être tellement populaire que, non seulement elle détourne notre attention d'autres phénomènes supposés beaucoup plus sérieux (tels que le style ou le contexte historique de l'œuvre), mais en plus, elle fait vendre des livres... elle est commerciale!

Cependant, ces dernières années, la réhabilitation de la lecture subjective et les études portant sur les lectures en progression, empiriques ou concrètes, nous obligent à reprendre, à nouveau frais, l'étude de cet effet esthétique autrefois négligé. Je tenterai par conséquent de montrer que s’intéresser aux dispositifs 
narratifs qui servent à produire, à accentuer ou, au contraire, à désamorcer la tension narrative, nous conduit à repenser l'articulation entre lectures du "premier degré» et lectures du «second degré». Plutôt que de culpabiliser les lecteurs qui lisent pour l'intrigue, et de disqualifier d'emblée tout ce qui se rattache dans l'œuvre à ce que Barthes appelait le "strip-tease narratif", on pourrait inviter ces apprenants à s'interroger sur les moyens poétiques qui érotisent leur lecture. Entrer dans la fabrique de l'intrigue, ce serait l'occasion de prendre conscience de la grande complexité de ces dispositifs narratifs, et de découvrir qu'il n'y a pas lieu de les considérer avec dédain comme de "grosses ficelles» ou des procédés "cousus de fil blanc». Il s'agit aussi de découvrir que la tension narrative n'est pas réservée aux seuls romans populaires, et que certaines œuvres, qui semblent pourtant assez expérimentales, ont trouvé des voies inédites pour nouer des intrigues efficaces ${ }^{\mathrm{I}}$. Enfin, on peut espérer que, dans une société où, pour le meilleur ou pour le pire, le storytelling prend de plus en plus de place, la didactisation de la tension narrative pourrait contribuer à développer chez le lecteur une certaine autonomie critique vis-à-vis des effets programmés par les textes, voire à envisager de passer du rôle passif de sujet lecteur, au rôle actif de sujet scripteur.

\section{La tension narrative, qu'est-ce que c'est?}

La tension narrative est une notion théorique qui vise à redéfinir le fonctionnement de l'intrigue sur un plan dynamique. Je la considère personnellement comme un hyperonyme recoupant différents effets que l'on associe ordinairement aux récits de fiction pourvus d'une intrigue : notamment le suspense, la curiosité et la surprise. Ces modalités de la tension narrative renvoient en elles-mêmes à la manière dont une intrigue se noue ou se dénoue, formant ainsi des séquences qui rythment la représentation narrative. L'intrigue, lorsqu'on la considère non comme une configuration statique, mais plutôt comme une forme en mouvement, comme une transformation dont la nature fondamentale est d'introduire et, éventuellement, de résoudre une tension, ressemble davantage à un labyrinthe qu'à une belle architecture classique, symétrique et bien ordonnée.

I. À ce sujet, je me permets de renvoyer à mon dernier ouvrage, et notamment aux chap. 2 et 3, qui traitent de l'intrigue dans les romans de Gracq : L'Euvre du temps, Paris, Seuil, coll. "Poétique», 2009. Voir aussi : La Tension narrative, Paris, Seuil, coll. «Poétique», 2007, et "Une mise à jour des outils narratologiques pour l'analyse de la littérature», Études de Lettres, $\mathrm{n}^{\circ} 4$, 2007, p. $175-205$. 
L'intrigue ménage des surprises lorsqu'elle s'écarte du chemin attendu.

Elle induit du suspense lorsqu'elle raconte des événements importants et dont le développement reste en partie indéterminé.

Elle suscite de la curiosité lorsque ces événements deviennent difficiles à interpréter, lorsqu'ils sont présentés de manière incomplète ou mystérieuse.

Ainsi, l'intrigue se noue lorsque nous hésitons à la croisée des chemins, lorsque le cours de l'histoire devient soudain imprévisible ou que sa nature devient mystérieuse, et cette tension nous invite à anticiper les virtualités d'un monde en mouvement. Si l'on considère l'intrigue durant le temps de sa réalisation, le dénouement apparaît dès lors comme une fonction secondaire, comme l'un des avenirs possibles de la narration, et non comme son fondement ${ }^{2}$. Pour comprendre la dynamique de l'intrigue, il est essentiel de tenir compte à la fois de la fin effective du texte et de ses fins possibles, des structures textuelles inscrites dans le récit mais aussi d'un acte de lecture qui articule les structures actualisées avec des structures actualisables, des histoires qui ont un mode d'existence virtuel, potentiel ou alternatif.

Par ailleurs, l'intrigue présuppose un narrateur intriguant qui s'adresse à un narrataire intrigué3. En d'autres termes, la tension qui noue l'intrigue doit se penser dans le cadre d'une interaction, et elle est d'abord un désordre provisoire dans la transmission d'un message. Un récit bien noué, c'est un récit qui refuse d'aller droit au but et qui préfere au contraire se perdre dans un jardin aux sentiers qui bifurquent, avant de livrer une information que le lecteur attendait avec impatience. Dans le contexte ludique de la fiction ${ }^{4}$, chaque partenaire de l'interaction est censé accepter de «jouer le jeu de l'intrigue». Cela implique, par exemple, que l'auteur prendra soin de tenir en haleine son lecteur, tandis que ce dernier se gardera de lire par avance la fin du roman, de respecter, au moins en partie, sa linéarité, de manière à ne pas gâcher la tension. Ainsi, la réticence de la représentation fictionnelle, qui serait horripilante dans d'autres contextes interactionnels, se transforme en un plaisir exaltant, comme une partie d'échecs ou de poker, comme un jeu du chat et de la souris.

2. L'intrigue n'est d'ailleurs pas toujours élaborée sur un mode déterministe et rétrospectif. Ainsi que l'a montré $\mathrm{M}$. Escola, le modèle de causalité rétrograde associé à l'intrigue, notamment tel qu'il est exposé par E. Poe dans sa fameuse "Genèse d'un poème», n'a que peu de pertinence pour décrire le mode de formation des intrigues romanesques du XviII ${ }^{\mathrm{e}}$ siècle. On peut en dire autant des feuilletons, des Mystères de Paris aux Soprano.

3. Évidemment, je prends ces termes dans un sens beaucoup plus général que leur sens technique. Il ne s'agit pas d'instances narratives construites par le discours, mais bien du producteur réel du récit et de ses destinataires.

4. Sur cette question, je renvoie naturellement à J.-M. Schaeffer, Pourquoi la fiction?, Paris, Seuil, coll. «Poétique», I999. 
Dans ce contexte spécifique, on ne lit donc pas pour le dénouement, pour le plaisir de savoir ou pour mettre de l'ordre dans le chaos de l'expérience, mais bien pour faire l'expérience délectable (parce que sans dangers et sans enjeux sérieux) du désordre, de l'étrangeté ou de la discordance qui précèdent le retour à l'ordre. Ainsi que l'affirmait Aristote, la valeur du pathos est dès lors inversée et ce qui nous affecterait dans notre vie quotidienne devient un spectacle, une source de jouissance, comme lorsque nous jouons à nous faire peur sur des montagnes russes.

Cette conception de l'intrigue, que je ne fais que résumer rapidement, n'est certes pas nouvelle. Elle trouve son origine dans l'analyse par Aristote de la catharsis. Dans un contexte plus récent, elle se rattache également à la tradition fonctionnaliste ou rhétorique, illustrée notamment par les travaux de Meir Sternberg's ou de James Phelan ${ }^{6}$. On peut mentionner également l'approche psychanalytique de Peter Brooks ${ }^{7}$, qui associe la dynamique de l'intrigue à une véritable érotique de la lecture, ou l'approche cognitiviste de Marie-Laure Ryan $^{8}$. Si la tension narrative a donc fait l'objet d'analyses approfondies dans le champ de la théorie du récit, et si elle tend à s'imposer dans le champ de la didactique`, on constate cependant qu'elle a encore du mal à entrer dans les pratiques enseignantes, et cela, à mon avis, pour deux raisons principales.

Premièrement, on peut se poser la question de savoir s'il est possible de légitimer, dans un enseignement de la littérature, non seulement l'intérêt des œuvres ou des dispositifs narratifs qui ont pour but d'intriguer les lecteurs, mais aussi, et surtout, l'intérêt de la lecture intriguée, de cette lecture passionnée qui progresse en tâtonnant vers un dénouement incertain.

Deuxièmement, pour appliquer un tel cadre d'analyse, il devient nécessaire de redéfinir certains concepts narratologiques fondamentaux dont la définition passait pour acquise, notamment le concept, hautement polysémique, d'intrigue. Il s'agit aussi de repenser le sens que l'on donne à l'analyse du «discours du récit ${ }^{10}$ », en la réarticulant avec la question de la séquence narrative et de

5. M. Sternberg, "Telling in Time (II): Chronology, Teleology, Narrativity», Poetics Today, $\mathrm{n}^{\circ}$ I3, I992, p. 463-54I.

6. J. Phelan, Reading People, Reading Plots: Character, Progression, and the Interpretation of Narrative, Chicago, University of Chicago Press, 1989.

7. P. Brooks, Reading for the Plot: Design and Intention in Narrative, Cambridge-Londres, Harvard University Press, 1984.

8. M.-L. Ryan, Possible Worlds, Artificial Intelligence, and Narrative Theory, Bloomington, Indiana University Press, I99I.

9. Ainsi que me l'a signalé N. Rannou, l'intérêt accordé à la tension narrative, même si elle n'est pas ainsi nommée, a motivé l'introduction de la lecture cursive dans les programmes de 2002 en France.

Io. G. Genette, Discours du récit, Paris, Seuil, coll. «Points Essais», 2007. 
l'intérêt romanesque. En effet, on verra que l'analyse de la tension narrative exige de passer du "comment» au "pourquoi», d'une simple description des structures à une interprétation de leur finalité esthétique.

J'espère arriver à montrer que, si l'on parvenait à surmonter ces difficultés, la didactique de la littérature trouverait de nombreux avantages à adopter un tel cadre d'analyse. Il ne s'agit évidemment pas de substituer l'analyse de la tension narrative à l'interprétation culturelle, historique, stylistique ou éthique de l'œuvre. Il est tout simplement question d'ajouter une corde supplémentaire à l'arc de l'enseignant, d'enrichir l'étude des romans en reprenant au sérieux, à l'instar d'Aristote, les effets esthétiques qui intéressent au premier plan les lecteurs ordinaires, avant que l'œuvre ne devienne un objet d'étude. La lecture en classe serait ainsi pensée non comme une négation de la lecture privée, mais comme son prolongement et sa fructification.

\section{De l'intérêt d'étudier le premier degré de la lecture}

Je vais donc commencer par tenter de définir, puis de justifier, l'intérêt de cette forme de lecture à laquelle il est possible d'associer la dynamique de la tension narrative. La plupart des théories de la lecture proposent des typologies définissant différentes catégories de lecteurs, ou différentes postures qu'un même lecteur est susceptible d'adopter, que ce soit successivement ou conjointement. $\mathrm{Au}$ sein de ces typologies, on retrouve généralement une dichotomie dans laquelle s'oppose une lecture première ou naïve, progressant rapidement dans le texte et soumise à l'intrigue, à une forme d'actualisation de l'œuvre beaucoup plus distanciée, critique, échappant aussi bien à l'illusion référentielle qu'à la linéarité du récit. Bertrand Gervais, parmi d'autres, a ainsi décrit la «lecture littéraire» comme un cheminement en deux temps :

Après un premier passage heuristique, qui correspond à une première saisie et interprétation (sommaire, fonctionnelle) du texte, le lecteur y revient pour en approfondir la connaissance, pour opérer un nouveau passage, inscrit sous le signe cette fois de l'herméneutique ${ }^{\text {II }}$.

Gervais oppose ainsi la «lecture en progression » à la «lecture en compréhension" et définit la «lecture littéraire» comme le passage d'un mode d'appropriation à l'autre.

Claude Lafarge relevait pour sa part que la lecture «légitime» nécessitait la «mise à distance du récit qui "piège" l'attention du lecteur incompétent ${ }^{12}$ ». Et

II. B. Gervais, «Lecture : tensions et régies", Poétique, n 89, I992, p. I05-I25, p. I22.

I2. C. Lafarge, La Valeur littéraire. Figuration littéraire et usages sociaux des fictions, Paris, Fayard, 1983, p. 215 . 
il insistait particulièrement sur la logique de distinction sociale qui se fonde sur cette opposition entre "lecteurs dominés», soumis aux artifices de la littérature de masse, et «lecteurs dominants», qui sont capables de s'en libérer. On peut associer à cette lecture "illégitime», dont parle Lafarge, les effets d'une intrigue qui soumet le lecteur à ses charmes. Ainsi que le résume Johanne Villeneuve :

Certains voient [dans l'amateur d'intrigues] un pur consommateur, un entiché de mécanique, un féru de scandale, un boulimique livré vivant à la production en chaîne de la culture de masse, aveugle à la complexité des formes et sourd à l'appel de la poésie. Ainsi tournée à la caricature, l'intrigue aurait force et pouvoir d'aliénation : elle tiendrait le spectateur naïf en haleine, le rive à ses fantasmes comme à son siège ${ }^{13}$.

Jean-Louis Dufays ${ }^{14}$ a pour sa part approfondi cette dichotomie en insistant sur l'existence d'un double système de valeurs qui se rattachent à ces deux formes d'appropriation du texte :

\begin{tabular}{|c|c|}
\hline \multicolumn{2}{|c|}{ LA DIALECTIQUE DES VALEURS } \\
\hline $\begin{array}{c}\text { Valeurs «fonctionnelles» } \\
\text { «classiques» et «dominées» }\end{array}$ & $\begin{array}{c}\text { Valeurs «littéraires» } \\
\text { «modernes» et «dominantes» }\end{array}$ \\
\hline Correction linguistique & Transgression linguistique \\
\hline Richesse du contenu & «Beauté», richesse des formes \\
\hline Réalité ou vraisemblance & Fictionnalité \\
\hline Moralité, bonté & Transgression éthique \\
\hline Unité, clarté & Polysémie, complexité \\
\hline Conformité & Subversion, nouveauté \\
\hline Émotion & Impassibilité \\
\hline \multicolumn{2}{|c|}{ Tension et va-et-vient entre les deux polarités } \\
Conception contemporaine («postmoderne») de la littérarité \\
\hline
\end{tabular}

Plus récemment, Jérôme David est venu rappeler qu’à côté de la lecture du «second degré», à la fois lecture critique et lecture valorisée par l'institution, il existait une «lecture de premier degré», beaucoup plus désintéressée, dans

13. J. Villeneuve, Le Sens de l'intrigue, Québec, Presses universitaires Laval, 2003, p. I3.

I4. J.-L. Dufays, «Lire, c'est aussi évaluer. Autopsie des modes de jugement à l'œuvre dans diverses situations de lecture", ÉLA, Revue de didactologie des langues-cultures, ${ }^{\circ}$ II9, 2000, p. $277-290$, p. 288. 
laquelle l'intérêt essentiel consistait à «s'identifier aux personnages et vibrer pour leurs malheurs ou leurs joies, les prendre pour modèles de conduite ou pour contre-exemples, se dépayser dans l'univers exotique de la fiction, être diverti par une intrigue palpitante ${ }^{\mathrm{I}}{ } »$. Jérôme David souligne que cette lecture a toujours été perçue comme une menace pour la société, tout comme aujourd'hui on s'inquiète des méfaits des jeux vidéo, de la bande dessinée et de la télévision, qui pervertiraient une jeunesse vouée à la déperdition morale et à l'analphabétisme. Ainsi, les institutions morales, telles que l'église ou l'école, se sont-elles donné pour mission d'éduquer les lecteurs, de les inviter à passer d'un mode de lecture à un autre :

Grand Partage des lectures : la lecture savante contre la lecture ordinaire; le dévoilement contre la duperie; la saisie contre la passivité; la pénétration virile contre l'accueil féminin... Spectres de Cervantès, en l'occurrence, ou de Fénelon (celui de L'Éducation des filles), ou de Stendhal, ou de Balzac, ou de Flaubert. Autant de traces indélébiles des craintes qui ont ponctué l'histoire de la discipline imposée à l'expérience esthétique par l'Église, l'École ou la bienséance, lorsque les femmes, les enfants ou les pauvres se sont mis à lire ${ }^{16}$.

L'institution scolaire serait donc également une école de la lecture «disciplinée». Il ne s'agit pas simplement d'apprendre à lire un roman, car cela, tout le monde est capable le faire dès lors qu'il sait déchiffrer l'écriture, que ce soit dans le train, sur la plage, ou caché sous ses draps avec une lampe de poche. Mais il s'agit de lire autre chose et autrement, de cesser de lire des livres faciles de manière frivole. On nous apprend à découvrir les classiques et à les décortiquer, à les soumettre à la question, à sortir de l'illusion de la fiction, à prendre le style au sérieux. On apprend, pour gagner de la distinction, à désirer des romans difficiles et austères, forcément dénués de suspense, de rebondissements ou d'intrigue. Pourvus d'une telle éducation, c'est avec surprise que nous découvrons un jour, presque par hasard, que certains monuments canonisés par l'institution ne sont pas aussi ennuyeux que ce que l'on aurait pu craindre. C'est du moins le témoignage que nous livre Frank Wagner, qui revient sur sa découverte de l'œuvre de Robbe-Grillet :

C'est durant les années 80, sur les conseils avisés d'un professeur de classe préparatoire, que je me suis hasardé à la découverte de l'œuvre d'Alain Robbe-Grillet. Hasardé, car mes camarades et moi-même avions été dûment mis en garde. Il s'agissait certes d'une œuvre importante, mais non moins exigeante : expérimentale, complexe, à certains égards aride, voire rebutante. [...] Près de 25 ans après, je me souviens encore de cette heureuse surprise : en lieu et place du pensum

I5. J. David, «Le premier degré de la littérature» [en ligne], $L H T, \mathrm{n}^{\circ} 9,20 \mathrm{I2}$. Disponible sur $<$ http://www.fabula.org/lht/9/index.php?id=304>, paragr. I8.

I6. Ibid., paragr. 8. 
redouté, une expérience de lecture en tous points stimulante, sur le triple plan cérébral, imaginaire et affectif. Mais, pour être honnête, autant voire davantage que de la construction savante du roman, ou que de ses notoires morceaux de bravoure «chosistes» (Ah! la description du quartier de tomate dans le restaurant automatique...), ce bonheur de lecture découlait de l'emprise fascinante exercée par un univers fictionnel d'une remarquable densitér ${ }^{17}$.

Ainsi, en dépit des avertissements et des préjugés qui nous ont été inculqués, nous sommes étonnés de découvrir que des romans tels que Les Gommes, La Modification, ou L'Homme sans qualités ne sont pas dénués d'intérêt et se laissent malgré tout assez facilement lire. Nous sommes surpris de découvrir de l'élan dans un roman de Julien Gracq, alors qu'on nous avait pourtant avertis qu'il ne s'y passait pratiquement rien. Mais l'institution risque de nous faire revenir de notre surprise, et nous finissons par nous convaincre que ce plaisir était certainement étranger à l'intrigue ou à une forme quelconque d'immersion. Nous apprenons au contraire à glorifier la prétendue absence d'intrigue que l'on croit reconnaître à chaque coin de page puisque l'œuvre est consacrée.

À mon avis, nous avons tort de nous étonner, et nous avons tort de disqualifier d'emblée l'intrigue lorsque nous abordons ce que l'on appelle communément la littérature d'avant-garde. L'institution scolaire pourrait aussi avoir pour mission de nous montrer comment les œuvres les plus exigeantes de la modernité ont trouvé des formules inédites pour intéresser leurs lecteurs, pour produire des avatars inattendus de l'intrigue romanesque et de la tension narrative. Avec Gracq, on peut apprendre que l'attente fiévreuse de l'ennemi ou de son amante peut intriguer davantage que le récit d'un conflit ou d'une passion déchirante. Avec Butor ${ }^{18}$, qu'un simple voyage en train, sans déraillement et sans aventures particulières, peut devenir passionnant lorsque nous ignorons, dans un premier temps, la raison pour laquelle le passager est parti de chez lui et, dans un deuxième temps, s'il se tiendra à la décision essentielle qui l'a amené à accomplir ce déplacement.

De toute façon, les temps ont changé, et aujourd'hui, face au recul de la littérature dans nos pratiques culturelles, on se dit que lire Balzac pour son intrigue, et même lire Eugène Sue ou Gaboriau, ce n'est déjà pas si mal... c'est toujours mieux que de ne pas lire du tout. On se rappelle aussi le plaidoyer de Daniel Pennac dans Comme un roman, qui revendiquait pour le lecteur le droit au «bovarysme», c'est-à-dire le droit à la satisfaction immédiate et exclusive de

I7. F. Wagner, «Raconter est devenu proprement impossible. Fabula et intrigue dans l'œuvre romanesque de A. Robbe-Grillet» [en ligne], Atelier de théorie littéraire. Fabula, 2oIr. Disponible sur <http://www.fabula.org/atelier.php?Fabula_et_intrigue> [consulté le oI/I2/20I3].

18. M. Butor, «Le roman comme recherche», dans Essais sur le roman, Paris, Gallimard, I960. 
ses sensations ${ }^{19}$. On peut également être sensible à sa critique de l'institution scolaire, qu'il associe à une école du "désenchantement", entraînant le risque de détourner durablement la jeunesse de la littérature. Ainsi que l'affirme encore Jérôme David:

On peut se demander, en effet, si la justification des études littéraires ne passe pas, aujourd'hui, par la réconciliation de la «lecture savante» et de la «lecture courante». [...] Parce que la lecture "courante» ou «naïve» semble ne pas concerner seulement les autres, mais court-circuiter les clivages du savant et du populaire, du légitime et de l'illégitime, du sérieux et du frivole. Parce qu'enfin ce que les textes "font" à leurs lecteurs se déploie souvent d'abord au premier degré, si bien que l'interprétation des œuvres ne peut pas exclure a priori cette dimension du sens, au motif qu'elle fourmillerait de trompe-l'œil qui entravent l'accès à un second degré (ironique, critique, autotélique...) jugé plus crucial ${ }^{20}$.

Ce que l'on observe depuis une vingtaine d'années, c'est un certain regain d'intérêt pour la lecture au premier degré, ou du moins, sur un plan axiologique, c'est un rééquilibrage entre différentes formes d'appropriation ou de «consommation» du texte littéraire. Annie Rouxel, en se fondant sur des autobiographies de lecteurs, constate d'ailleurs que «l'identification est au cœur des souvenirs évoqués ou analysés par les jeunes aussi bien que par les adultes grands lecteurs ${ }^{21} »$, et cette identification apparait aussi comme une "expérience essentielle, hautement revendiquée par les lycéens comme source du plaisir de lire». Rouxel ménage ainsi, à côté d'autres instances lectoriales, critiques ou distanciées, une place pour un lecteur fugueur «qui envisage la littérature comme une évasion de soi et de la réalité dans un temps aboli ${ }^{22}$ ». Elle ajoute que pour ce lecteur, le «rythme de lecture est rapide, haletant : l'attention du sujet est portée sur l'intrigue dans la quête du dénouement". On pourrait aussi rattacher à la tension narrative et à l'intrigue une autre instance lectoriale définie par Rouxel : le lecteur spectateur, qui ressent «un mouvement d'empathie pour les personnages et suscite un voyage dans sa propre intériorité. Le lecteur, spectateur attentif, est à l'écoute de lui-même, aux aguets: il vit le texte ${ }^{23} »$.

Jouve affirme pour sa part que, pour le lecteur soumis à l'illusion référentielle, «la catharsis permettrait [...] d'assumer le refoulement social de ses

19. D. Pennac, Comme un roman, Paris, Gallimard, 1992.

20. J. David, «Le premier degré de la littérature», art. cité, paragr. 25.

2I. A. Rouxel, "Autobiographie de lecteur et identité littéraire», dans A. Rouxel et G. Langlade (dir.), Le Sujet lecteur. Lecture subjective et enseignement de la littérature, Rennes, Presses universitaires de Rennes, 2004, p. I37-I5I, p. I44.

22. Ibid., p. 146.

23. Ibid., p. I46. 
pulsions en les vivant par personnages interposés ${ }^{24} »$. Dans une perspective plus ludique, Picard ajoute que c'est dans l'équilibre entre un «jeu de rôle», dans lequel, littéralement, on se prend au jeu et un "jeu de société», dans lequel on est pleinement conscient des règles du jeu, que se situe l'expérience esthétique. Il affirme ainsi que «la qualité littéraire, la richesse du jeu et donc son efficacité [sont] fonction de ce double équilibre ${ }^{25}$ ». Quant à Jean-Louis Dufays, son schéma laisse déjà entrevoir que, dans l'esthétique postmoderne, les deux instances tendent vers un équilibre dynamique.

Jérôme David entrevoit pour sa part deux «pistes» qui permettraient de remettre à l'honneur la lecture du premier degré, aussi bien dans le cadre des études littéraires que dans celui de son enseignement :

La première [piste] nous conduit à théoriser ce que peut être l'expérience de lecture à partir de cas avérés - qu'il s'agisse de notre propre lecture ou de celle des autres. Comment un texte peut-il affecter son lecteur? [...] [La] seconde piste : [consisterait à] produire une théorisation de la littérature qui fasse d'emblée la part belle au premier degré de la littérature ${ }^{26}$.

Ainsi que le proposait récemment Marielle Macée ${ }^{27}$, la première piste pourrait nous amener à tenir compte des récits de quelques "grands lecteurs», dont on découvre qu'ils ne lisent pas toujours au second degré, et qu'ils savent aussi se laisser «affecter" par les récits qui les passionnent.

On pourrait aussi tenter de confronter les lectures du second degré que nous devons à des lecteurs professionnels, avec des lectures du premier degré, telles que nous pouvons les reconstruire à travers l'analyse de la réception critique d'une ouvre. Cette comparaison ferait certainement ressortir quelques contrastes saisissants et productifs. Ainsi, dans un commentaire de Derborence, Jean-Louis Pierre souligne que, dès l'épigraphe, qui révèle certains éléments de l'intrigue, Ramuz laissait entrevoir ses intentions : «l'essentiel ne [sera] pas l'“invention" d'une intrigue complexe, ou les effets d'intensité dramatique [...]. L'œuvre semble ainsi s'orienter dans la voie du poétique plutôt que du romanesque $^{28}{ }$ Ce commentaire, rédigé au moment où Derborence entre enfin

24. V. Jouve, L'Effet-personnage dans le roman, Paris, Presses universitaires de France, I992, p. 240.

25. M. Picard, «Lector ludens», Série Mutations, n ${ }^{\circ}$ I53, I995, p. I32-I44; M. Picard, La Lecture comme jeu : essai sur la littérature, Paris, Minuit, I986, p. I39.

26. J. David, «Le premier degré de la littérature», art. cité, paragr. 30, 34.

27. Voir son ouvrage Façon de lire, manières d'être, Paris, Gallimard, coll. "NRF Essais», $201 \mathrm{.}$ G. Langlade et A. Rouxel empruntaient déjà cette direction en 2004 dans Le Sujet lecteur. Lecture subjective et enseignement de la littérature, Rennes, Presses universitaires de Rennes, 2004.

28. C.-F. Ramuz, Euvres complètes, t. 2, Paris, Gallimard, coll. «Bibliothèque de la Pléiade», 2005 , p. 1665 . 
au panthéon des œuvres éditées dans la prestigieuse collection de la Pléiade, s'oppose pourtant clairement aux qualités esthétiques mises en évidence par la critique et adressées aux lecteurs ordinaires, lors de sa parution en France en 1936. En effet, à cette époque, nous pouvons lire que Derborence est un "drame poignant écrit dans un style elliptique qui laisse à chaque page une incertitude de plus en plus pesante jusqu'aux dernières lignes ${ }^{29}$ ». Ou encore : «Derborence qui paraît ces jours-ci, est d'un pathétique intense et déconcertant. Il prend tout de suite le lecteur, mais pour le dérouter, et ne pas le laisser se reprendre, tant que le roman n'est pas fini ${ }^{30}$.» On peut constater, à travers ce cas qui me parait exemplaire, le risque que le lecteur professionnel ou "éduqué" devienne incapable de reconnaitre le caractère palpitant d'un récit canonisé, alors que le phénomène de la tension narrative est pourtant largement attesté par les commentaires publiés du vivant de l'auteur.

Pour traiter la littérature contemporaine, et pour se rapprocher encore davantage des «lecteurs ordinaires», on pourrait aussi mentionner un nouvel outil qui s'offre à nous. Je pense notamment aux bases de données regroupant les avis de lecteurs non professionnels publiés sur les sites Internet de sociétés commerciales, tels qu'Amazon, par exemple. Dominique Legallois et Céline Poudat, en se basant sur ce genre de corpus, ont récemment mis en évidence le fait que les valeurs impactant le plus l'évaluation de l'œuvre étaient, sans surprise, "l'intrigue et [le] happage, et plus spécifiquement, [...] l'ensemble des valeurs émotives et morales ${ }^{3 \mathrm{I}} »$. Ils concluent ainsi que, pour les lecteurs ordinaires, l'intrigue est "un critère de première importance, qui détermine très significativement l'évaluation de l'œuvre; une intrigue évaluée comme pauvre entraîne ainsi une recommandation négative de l'œuvre ${ }^{32} »$.

\section{De la nécessité de faire évoluer les concepts narratologiques et leur usage}

Pour conclure, j'aimerais rapidement revenir sur la seconde piste évoquée par Jérôme David, à savoir la façon dont il faudrait théoriser le récit de manière à ce que l'on «fasse d'emblée la part belle au premier degré de la littérature».

29. Bulletin PLM, septembre 1936.

30. L'Européen, 20 mars 1936.

31. D. Legallois et C. Poudat, «Comment parler des livres que l'on a lus? Discours et axiologie des avis des Internautes" [en ligne], Semen, ${ }^{\circ}$ 26, 2008, paragr. I03. Disponible sur <http:// semen.revue.org/8444> [consulté le oI/II/20I3].

32. Ibid., paragr. Ioo. 
J'aimerais d'abord insister sur l'importance de tenir compte d'un problème de polysémie qui entoure la notion d'intrigue, et qui faisait récemment dire à Hilary Dannenberg, dans la très sérieuse Routledge Encyclopedia of Narrative Theory, que «malgré l'apparente simplicité de l'objet auquel elle se réfère, l'intrigue est l'un des termes les plus insaisissables de la théorie du récit ${ }^{33}$ ". Pour ma part, il me semble que réduire l'intrigue à la trame de l'histoire racontée est un appauvrissement conceptuel qui conduit à occulter la fonction de la mise en intrigue et sa dépendance envers les liens complexes qui se tissent entre la surface du discours et l'histoire racontée.

Le principal changement conceptuel au niveau du traitement de l'intrigue consiste donc à accepter de redéfinir le nœud, non pas en fonction d'une complication qui affecterait les personnages de l'histoire racontée, mais bien en fonction d'une complication informationnelle dans l'interaction entre le discours narratif et son lecteur. Le nœud n'est donc plus rattaché à la logique immanente de l'histoire racontée, mais à une réticence dans la narration qui conduit à attendre un dénouement. Lorsque, soudain, quelque chose arrive dans l'histoire, l'événement, dont les conséquences nous sont inconnues, engendre du suspense, mais pour cela, le récit doit respecter au moins en partie la chronologie de l'histoire. Dans le cas contraire, la logique de l'action devient un puzzle, et le lecteur est amené à s'interroger sur la manière de recomposer une suite cohérente d'événements à partir de ce désordre, ce qui débouche sur une manière alternative de nouer l'intrigue, beaucoup moins dépendante de la chronologie de l'histoire. Cela implique également qu'un récit se noue et se dénoue toujours dans l'ordre de son actualisation par un interprète, et non dans l'ordre de l'histoire racontée. En d'autres termes, un récit ne commence jamais par le dénouement, même s'il commence par narrer la résolution d'un conflit avant d'introduire ses causes éventuelles.

Par ailleurs, définir la dynamique de l'intrigue n'a rien à voir avec la tâche, relativement stérile, qui consiste à résumer et à hiérarchiser les événements principaux de l'histoire racontée. Au contraire, il s'agit de dénombrer les très nombreuses structures narratives qui auront pour fonction d'intriguer le lecteur, de construire son attente d'une suite plus ou moins inquiétante ou mystérieuse à partir d'une forme de "réticence» du texte. Il devient donc nécessaire de réconcilier l'analyse du discours narratif avec celle de sa séquentialité et de ses effets dynamiques sur le lecteur. Certes, raconter un conflit dont l'issue est incertaine sera probablement un bon moyen d'intéresser le lecteur et d'induire du suspense, mais respecter la chronologie de l'événement, ralentir l'écoulement

33. H. Dannenberg, "Plot», dans Routledge Encyclopedia of Narrative Theory, Londres, Routledge, 2005, p. 435. 
temporel ou procéder à un changement de focalisation de manière à renforcer l'identification avec la perspective du héros pourront être des éléments tout aussi essentiels.

Le simple surgissement d'un passé simple dans une nouvelle de Flaubert, au sein de laquelle toute forme de dramatisation était jusque-là écrasée par l'usage massif de l'imparfait, apparaîtra également comme un indice décisif de mise en intrigue, d'un procédé visant à créer de la tension narrative. Tout comme la description d'une atmosphère inquiétante, d'un cadre nocturne et nébuleux.

Un soir d'automne, on s'en retourna par les herbages. La lune à son premier quartier éclairait une partie du ciel, et un brouillard flottait comme une écharpe sur les sinuosités de Toucques ${ }^{34}$.

L'analyse axiologique des personnages deviendra également cruciale dans cette économie de la tension, pour autant que l'on résiste à la tentation de poser un jugement rétrospectif sur leurs rôles. On verra par exemple comment tel ou tel récit travaille à rendre difficile le rattachement d'un personnage au rôle actantiel de l'opposant ou de l'adjuvant. Ainsi en va-t-il du professeur Rogue dans la saga Harry Potter.

Sur un autre plan, l'analyse du point de vue permettra de souligner comment une restriction locale ou généralisée des informations accessibles au lecteur pourra servir à nouer un récit dès les premières lignes, par exemple en rendant énigmatique l'action d'un personnage inconnu. Ce que Genette appellerait une «focalisation externe" est un procédé très courant dans les incipit des romans de Balzac, par exemple dans le Cousin Pons, où il faut attendre une trentaine de pages, dans l'édition folio, pour comprendre qui est le protagoniste, où il se rend, quelle est la raison de sa satisfaction apparente, quel est l'objet qu'il cache sous son manteau et quel est le but de son déplacement. Les narrations homodiégétiques apparaissent aussi comme des scénographies qui permettent de jouer sur la restriction du savoir, ou sur ce qui pourrait pousser le narrateur à dissimuler des informations à son narrataire ${ }^{35}$.

Enfin, il est évident que la succession de passages singulatifs et itératifs, de scènes et de sommaires est étroitement corrélée à la succession de séquences plus ou moins tendues ou, au contraire, plates sur un plan dramatique. De même, la pause marque souvent un passage atone alors que l'ellipse ou la paralipse nous amènent généralement à nous interroger sur ce que le récit a passé sous silence. On pourra aussi montrer qu'une analepse ou une prolepse peuvent aussi bien servir à intriguer le lecteur, en brouillant le drame ou en retardant

34. G. Flaubert, Trois Contes, Paris, Le Livre de Poche, 1999, p. 55.

35. Je pense par exemple au narrateur du Meurtre de Roger Ackroyd de A. Christie. 
son développement, qu'à dénouer des mystères. L'analyse de la dynamique de l'intrigue permet ainsi de remotiver l'usage de l'ensemble des outils descriptifs de la narratologie, aussi bien thématique que modale.

J'aimerais terminer en insistant sur le fait qu'enseigner ces «techniques» de l'intrigue ne vise pas uniquement à revitaliser les études littéraires en rendant justice à la qualité esthétique des œuvres qui nous passionnent, ces œuvres dont l'intérêt se manifeste jusque dans nos pratiques de lecture ordinaires. D'une part, cet enseignement pourrait être l'occasion de passer du rôle de sujet lecteur à celui de sujet scripteur : en mettant en place des ateliers d'écriture, les apprenants peuvent imiter certaines techniques, se les approprier pour les recontextualiser dans des productions originales. D'autre part, le sujet lecteur connaissant les rouages de l'intrigue peut aussi devenir un sujet critique. Analyser aussi bien la force de l'intrigue que sa forme, c'est aussi l'occasion de nous mettre en garde contre le pouvoir du storytelling, qui ne se manifeste malheureusement pas uniquement dans le champ de la feintise ludique et de l'esthétique, mais également dans le discours de médias en mal d'audience, de publicitaires ou de politiciens sans scrupules qui veulent gagner notre adhésion en «fabriquant des histoires» et en "formatant nos esprits", pour reprendre la formule de Christian Salmon ${ }^{36}$.

Je crois qu'il est important d'admettre que les récits ont un réel pouvoir rhétorique, et que s'intéresser à la tension narrative, c'est aussi poser la question des fondements poétiques de cette rhétorique et de la manière dont les récits affectent leurs lecteurs, leurs auditeurs ou leurs spectateurs. À mon avis, la valeur essentielle de la littérature, et ce qui justifie la place de la fiction dans les sociétés humaines, c'est le caractère ouvertement assumé, et souvent réflexif, de ce jeu poétique qui engage la force du récit. Le récit de fiction est peut-être parfois commercial, mais il est en même temps gratuit, puisqu'il n'est au service d'aucune autre visée extérieure à lui-même. En fin de compte, je suis convaincu qu'à travers l'étude de la tension narrative, la littérature continuera à être ce «laboratoire du récit» évoqué par Michel Butor ${ }^{37}$, un lieu au sein duquel la narrativité, omniprésente dans nos vies, se manifeste sous sa forme la plus expérimentale : c'est-à-dire qu'elle s'offre à la fois comme un objet d'expériences empiriques (de lecture ou d'écriture) et comme l'une des manifestations les moins normatives et les plus universelles de la représentation narrative. Étudier la tension narrative, c'est aussi étudier comment répondre à la mise en intrigue élaborée par autrui.

36. C. Salmon, Storytelling, la machine à fabriquer des histoires et à formater les esprits, Paris, La Découverte, 2007.

37. M. Butor, «Le roman comme recherche», dans Essais sur le roman, ouvr. cité, p. 9. 